\title{
Ultrasubwavelength Ferroelectric Leaky Wave Antenna in a Planar Substrate-Superstrate Configuration
}

\author{
G. Lovat, ${ }^{1}$ P. Burghignoli, ${ }^{2}$ R. Araneo, ${ }^{1}$ and S. Celozzi ${ }^{1}$ \\ ${ }^{1}$ Department of Astronautical, Electrical and Energetic Engineering (DIAEE), Sapienza University of Rome, \\ Via Eudossiana 18, 00148 Roma, Italy \\ ${ }^{2}$ Department of Information Engineering, Electronics and Telecommunications, Sapienza University of Rome, \\ Via Eudossiana 18, 00148 Roma, Italy \\ Correspondence should be addressed to G. Lovat; giampiero.lovat@uniromal.it
}

Received 20 May 2014; Revised 14 August 2014; Accepted 15 August 2014; Published 31 August 2014

Academic Editor: Yingsong Li

Copyright (C) 2014 G. Lovat et al. This is an open access article distributed under the Creative Commons Attribution License, which permits unrestricted use, distribution, and reproduction in any medium, provided the original work is properly cited.

The possibility of achieving directive fan-beam radiation with planar Fabry-Pérot cavity antennas constituted by an upper ferroelectric thin film and a lower ground plane having ultrasubwavelength thickness is studied by means of a simple transverseequivalent-network approach and a cylindrical leakywave analysis, deriving simple design formulas. The performance of the proposed antenna is investigated in terms of power density radiated at broadside and directivity in the principal planes, pointing out the main limitations and tradeoffs associated with the reduced thickness.

\section{Introduction}

Planar antennas based on partially reflecting surfaces (PRSs) have been receiving increasing interest during the last decade, thanks to their attractive features of low profile, high directivity, and simple feeding. Since the first design proposed and realized by von Trentini in 1956 [1], in which the directivity of truncated waveguides opening in an infinite conducting ground plane was increased by means of periodic metal screens, a number of different PRS realizations have been proposed, based, for example, on dielectric substrate-superstrate configurations [2] or on two-dimensional periodic array of metal patches $[3,4]$ or slots cut in a top plate [5].

The typical thickness of PRS-based planar antennas is of the order of one-half of the free-space wavelength. In fact, the directivity enhancement afforded by the PRS can be attributed to the resonant behavior of the partially open planar cavity formed by the ground plane and the PRS; the antenna can thus be viewed as a sort of Fabry-Pérot resonator (hence the name Fabry-Pérot cavity (FPC) antennas) [2, 6]. Alternatively, the antenna can be viewed as a twodimensional leaky wave antenna, in which the primary source excites a pair of cylindrical $\mathrm{TM}^{\mathrm{Z}}$ and $\mathrm{TE}^{\mathrm{Z}}$ leaky modes; these propagate along the structure and give the main contribution to the antenna aperture field, thus establishing the main features of the antenna radiation pattern $[7,8]$. A leaky wave analysis of FPC antennas showed that the power density radiated at broadside is maximum when the thickness of the cavity is optimum, that is, close to half of the wavelength inside the substrate above which the PRS is placed; when such an optimum thickness is used to create a broadside beam, the leaky modes supported by the structure have nearly the same wavenumber with nearly equal low values of the phase and attenuations constants [9-11] thus producing a highly directive broadside beam.

In this paper we study PRS-based planar antennas in which the total thickness is considerably smaller than the values typical of the usual FPC antennas $[2,7]$ and which has been the subject of many investigations [12-14]. A preliminary investigation on this kind of structure has been presented in [15]. Here we consider a planar substrate constituted by a conventional dielectric covered by a thin ferroelectric film. The structure is similar to that presented in [16] where the ferroelectric film was used to allow for an electrical tuning of the main parameters characterizing the radiation pattern of the antenna. Here the ferroelectric film 
is used to obtain an equivalent capacitive PRS which allows for dramatically reducing the total thickness of the antenna capable of obtaining a directive beam. The performance of the proposed antenna is investigated in terms of power density radiated at broadside and directivity in the principal planes, pointing out the main limitations and tradeoffs associated with the reduced thickness.

\section{Structure Description}

The reference structure, shown in Figure 1, is a dielectric slab (the substrate) of thickness $b$ and relative permittivity $\varepsilon_{r 1}$, bounded below by a perfectly conducting (PEC) ground plane and covered by a ferroelectric film of thickness $t$ with permittivity $\varepsilon_{r 2}=\varepsilon_{r 2}^{\prime}-j \varepsilon_{r 2}^{\prime \prime}$. The materials are assumed to be nonmagnetic (i.e., $\mu_{r 1}=\mu_{r 2}=1$ ). The structure is assumed to be excited by a unit-amplitude $(1 \mathrm{~V} \cdot \mathrm{m})$ horizontal magnetic dipole (HMD) directed along the $x$-axis and placed over the ground plane, thus modeling a thin slot etched on the ground plane and excited by a suitable waveguide structure (e.g., a microstrip line). This kind of source is chosen instead of the more conventional horizontal electric dipole (HED) since in an ultrasubwavelength configuration the latter would radiate poorly due to the electrical closeness to the PEC ground plane. A time-harmonic dependence $\exp (j \omega t)$ of sources and fields will be assumed and suppressed throughout.

\section{Antenna Radiation}

3.1. Far-Field Pattern via Reciprocity. The far-field pattern radiated by the HMD in the presence of the planar structure described above can be calculated through a standard application of reciprocity theorem, by letting a plane wave impinge on the structure from the observation direction $(\theta, \phi)$ and calculating the reaction between the resulting total field and the HMD source $[2,6]$. The field produced by the incident plane wave can in turn be readily calculated using the transverse equivalent network (TEN) representation of the structure shown in Figure 2(a), where the substrate and superstrate layers are modeled with lengths of transmission lines (TLs).

Assuming that the relative permittivity of the ferroelectric film is very large $\left(\left|\varepsilon_{r 2}\right| \gg 1\right), k_{z 2}=k_{0} \sqrt{\varepsilon_{r 2}-\sin ^{2} \theta} \cong k_{z 2}=$ $k_{0} \sqrt{\varepsilon_{r 2}}$ results and hence $Y_{2} \cong \sqrt{\varepsilon_{r 2}} / \eta_{0}$ for both TM and TE polarizations (here $k_{0}=2 \pi / \lambda_{0}$ and $\eta_{0}$ are the free-space wavenumber and characteristic impedance, resp.). Assuming further that the electrical thickness of the ferroelectric film is small, the relevant length of TL can be replaced by a shunt admittance $Y_{f}=G_{f}+j B_{f}=k_{0} t \varepsilon_{r 2} / \eta_{0}$ for both $\mathrm{TM}$ and TE polarizations $[16,17]$, thereby obtaining the TEN in Figure 2(b). Using the latter TEN, the expressions of the farfield pattern are obtained as

$$
\begin{aligned}
& E_{\theta}(r, \theta, \phi) \\
& \quad=E_{0} \sin \phi \frac{-2 j Y_{1}^{\mathrm{TM}}}{\left(Y_{0}^{\mathrm{TM}}+Y_{f}\right) \sin \left(k_{z 1} b\right)-j Y_{1}^{\mathrm{TM}} \cos \left(k_{z 1} b\right)},
\end{aligned}
$$

$$
\begin{aligned}
& E_{\phi}(r, \theta, \phi) \\
& =-E_{0} \cos \phi \cos \theta \frac{-2 j Y_{1}^{\mathrm{TE}}}{\left(Y_{0}^{\mathrm{TE}}+Y_{f}\right) \sin \left(k_{z 1} b\right)-j Y_{1}^{\mathrm{TE}} \cos \left(k_{z 1} b\right)},
\end{aligned}
$$

where

$$
E_{0}=-j k_{0} \frac{e^{-j k_{0} r}}{4 \pi r}
$$

and the TL parameters are

$$
\begin{aligned}
Y_{0}^{\mathrm{TM}} & =\frac{k_{0}}{\eta_{0} k_{z 0}}, \\
Y_{0}^{\mathrm{TE}} & =\frac{k_{z 0}}{\eta_{0} k_{0}} \\
Y_{1}^{\mathrm{TM}} & =\frac{\varepsilon_{r 1} k_{0}}{\eta_{0} k_{z 1}}, \\
Y_{1}^{\mathrm{TE}} & =\frac{k_{z 1}}{\eta_{0} k_{0}} \\
k_{z 0} & =k_{0} \cos \theta, \\
k_{z 1} & =\sqrt{\varepsilon_{r 1} k_{0}^{2}-\sin ^{2} \theta} .
\end{aligned}
$$

3.2. Design Equations for Maximum Broadside Radiation. From (1), at broadside we have

$$
\begin{aligned}
\left.E_{\phi}(\theta=0)\right|_{H} & =\left.E_{\theta}(\theta=0)\right|_{E} \\
& =E \\
& =E_{0} \frac{-2 j n_{1}}{\left(1+\bar{Y}_{f}\right) \sin \left(2 \pi n_{1} \widehat{b}\right)-j n_{1} \cos \left(2 \pi n_{1} \widehat{b}\right)},
\end{aligned}
$$

where $n_{1}=\sqrt{\varepsilon_{r 1}}, \widehat{b}=b / \lambda_{0}$, and $\bar{Y}_{f}=\eta_{0} Y_{f}$. For very thin substrates (i.e., $n_{1} \widehat{b} \ll 1$ ), (4) can be approximated using a Taylor expansion of the trigonometric functions as

$$
\begin{aligned}
E & \simeq E_{0} \frac{-2 j n_{1}}{\left(1+\bar{Y}_{f}\right)\left(2 \pi n_{1} \widehat{b}\right)-j n_{1}\left[1-\left(\left(2 \pi n_{1} \widehat{b}\right)^{2} / 2\right)\right]} \\
& \simeq \frac{-2 j E_{0}}{\left(1+\bar{G}_{f}\right) 2 \pi \widehat{b}+j\left(2 \pi \widehat{b} \bar{B}_{f}-1\right)} .
\end{aligned}
$$

Then, for a high-permittivity low-loss ferroelectric medium [18-20], $\bar{B}_{f} \gg \max \left\{1, \bar{G}_{f}\right\}$ results and the absolute value of the expression in (5) is maximized when $2 \pi \widehat{b} \bar{B}_{f}-1=0$; that is,

$$
\bar{B}_{f}=\frac{1}{2 \pi \widehat{b}}
$$




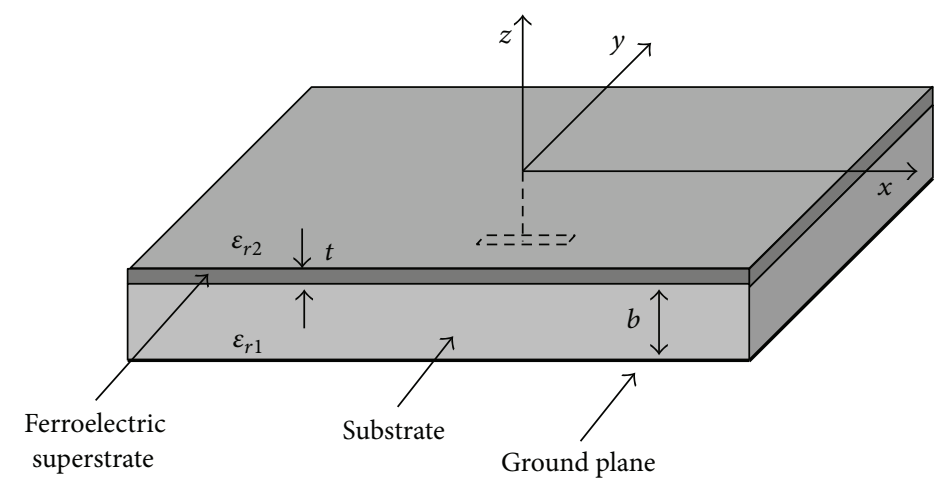

FIgURE 1: Planar substrate-superstrate configuration excited by a magnetic dipole over the ground plane with the relevant geometric and physical parameters.

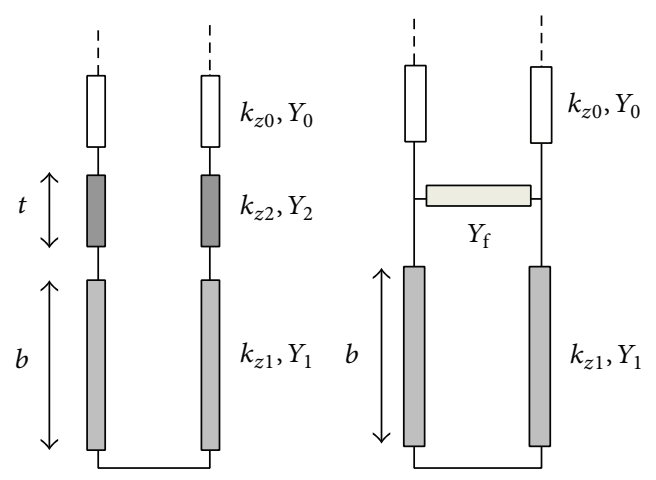

(a)

(b)

FIGURE 2: Transverse equivalent network of the structure in Figure 1. Exact representation (a) and approximate representation (b) valid for a high-permittivity thin-film superstrate.

or

$$
\widehat{b}=\frac{1}{2 \pi \bar{B}_{f}} .
$$

Therefore, very thin substrates require large values of the normalized (capacitive) susceptance $\bar{B}_{f}$. In such a case, the power density at broadside is given by

$$
P(0)=\frac{|E|^{2}}{2 \eta_{0}} \simeq \frac{k_{0}^{2}}{8 \pi^{2} \eta_{0}} \frac{\bar{B}_{f}^{2}}{\left(1+G_{f}\right)^{2}} \simeq \frac{k_{0}^{2}}{8 \pi^{2} \eta_{0}} \bar{B}_{f}^{2} .
$$

Therefore, the power density radiated at broadside is enhanced of a factor $\bar{B}_{f}^{2}$ with respect to the case of a magnetic dipole radiating on a PEC ground plane with free space above. (This may be contrasted with the case of a HED excitation [15], in which no such enhancement is found because the short-circuiting effect of the PEC ground plane compensates the resonant effect of the PRS.) To establish whether the enhancement of power density is accompanied with an enhancement of directivity as well, we have to determine if the structure can support leaky waves with small values of the phase and attenuation constants.

\section{Leaky Wave Analysis}

The dispersion equation coincides with the denominator in (1); that is, (neglecting $\bar{G}_{f} \ll \bar{B}_{f}$, [18-20])

$$
\bar{Y}_{0}^{\mathrm{TE} / \mathrm{TM}}+j \bar{B}_{f}-j \bar{Y}_{1}^{\mathrm{TE} / \mathrm{TM}} \cot \left(2 \pi \widehat{k}_{z 1} \widehat{b}\right)=0 .
$$

We assume a solution for the normalized longitudinal propagation constant $\widehat{k}_{x}$ of the form

$$
\widehat{k}_{x}=\delta=\delta_{R}-j \delta_{J}
$$

where $\delta_{R}$ and $\delta_{J}$ are "small" $\left(\left|\delta_{R}\right| \ll 1\right.$ and $\left.\left|\delta_{J}\right| \ll 1\right)$, so that the normalized transverse propagation constants $\widehat{k}_{z 0}$ and $\widehat{k}_{z 1}$ in air and inside the slab, respectively, are approximated as

$$
\begin{aligned}
& \widehat{k}_{z 0}=\sqrt{1-\widehat{k}_{x}^{2}}=\sqrt{1-\delta^{2}} \simeq 1-\frac{\delta^{2}}{2}, \\
& \widehat{k}_{z 1}=\sqrt{\varepsilon_{r 1}-\widehat{k}_{x}^{2}}=\sqrt{\varepsilon_{r 1}-\delta^{2}} \simeq n_{1}-\frac{\delta^{2}}{2 n_{1}}
\end{aligned}
$$


while the cotangent function in (9) can be approximated as

$$
\begin{aligned}
\cot \left(2 \pi \widehat{k}_{z 1} \widehat{b}\right) & \simeq \frac{\left(1 / 2 \pi n_{1} \widehat{b}\right)\left(-\left(n_{1} / \pi \hat{h} \delta^{2}\right)\right)-1}{\left(1 / 2 \pi n_{1} \widehat{b}\right)+\left(-\left(n_{1} / \pi \widehat{b} \delta^{2}\right)\right)} \\
& =\frac{n_{1}\left(1+2 \pi^{2} \widehat{b}^{2} \delta^{2}\right)}{\pi \widehat{b}\left(2 n_{1}^{2}-\delta^{2}\right)} .
\end{aligned}
$$

Now we have to consider separately the two cases of TE and TM modes.

4.1. TE Leaky Waves. For TE modes the transverse characteristic impedances are

$$
\bar{Y}_{0}^{\mathrm{TE}}=\widehat{k}_{z 0} \quad \bar{Y}_{1}^{\mathrm{TE}}=\widehat{k}_{z 1}
$$

Based on the above approximations, (9) can be written as

$$
1-\frac{\delta^{2}}{2}+j \bar{B}_{f}-j\left(n_{1}-\frac{\delta^{2}}{2 n_{1}}\right) \frac{n_{1}\left(1+2 \pi^{2} \widehat{b}^{2} \delta^{2}\right)}{\pi \widehat{b}\left(2 n_{1}^{2}-\delta^{2}\right)}=0 .
$$

That is,

$$
\begin{aligned}
\pi \widehat{b}\left(2 n_{1}^{2}-\delta^{2}\right)-\pi \widehat{b}\left(2 n_{1}^{2}-\delta^{2}\right) \frac{\delta^{2}}{2} \\
+j \pi \widehat{b}\left(2 n_{1}^{2}-\delta^{2}\right) \bar{B}_{f}-j\left(n_{1}-\frac{\delta^{2}}{2 n_{1}}\right) \\
\quad \times n_{1}\left(1+2 \pi^{2} \widehat{b}^{2} \delta^{2}\right)=0 .
\end{aligned}
$$

By neglecting all the terms $o\left(\delta^{4}\right),(15)$ is written as

$$
\begin{aligned}
& \left(4 n_{1}^{2} \pi \widehat{b}+j 4 n_{1}^{2} \pi \widehat{h} \bar{B}_{f}-j 2 n_{1}^{2}\right) \\
& \quad+\left(j-\pi \widehat{b}-2 n_{1}^{2} \pi \widehat{b}-j 2 \pi \widehat{h} \bar{B}_{f}-j 4 n_{1}^{2} \pi^{2} \widehat{b}^{2}\right) \delta^{2} \\
& =0 .
\end{aligned}
$$

Under the optimum condition (4) (or (5)), (16) becomes

$$
\left(2 n_{1}^{2} \frac{1}{\bar{B}_{f}}\right)+\left(-\frac{1}{2 \bar{B}_{f}}-n_{1}^{2} \frac{1}{\bar{B}_{f}}-j n_{1}^{2} \frac{1}{\bar{B}_{f}^{2}}\right) \delta^{2}=0 .
$$

That is,

$$
\delta^{2}=\frac{4 n_{1}^{2}}{\left(2 n_{1}^{2}+1\right)}-j \frac{8 n_{1}^{4}}{\left(2 n_{1}^{2}+1\right)^{2} \bar{B}_{f}} .
$$

By equating the real and the imaginary parts of (18) we obtain

$$
\begin{aligned}
& \delta_{R}^{2}-\delta_{J}^{2} \simeq \frac{4 n_{1}^{2}}{\left(2 n_{1}^{2}+1\right)} \\
& 2 \delta_{R} \delta_{J} \simeq \frac{8 n_{1}^{4}}{\left(2 n_{1}^{2}+1\right)^{2} \bar{B}_{f}} .
\end{aligned}
$$

Equations (19) contradict the assumption of "small" $\delta_{R}$ and $\delta_{J}$, so that no TE leaky waves exist in this case with $\widehat{\beta}=\widehat{\alpha}$ and $\widehat{\alpha} \ll 1$.
4.2. TM Leaky Waves. For TM modes the transverse characteristic impedances are

$$
\bar{Y}_{0}^{\mathrm{TM}}=\frac{1}{\hat{k}_{z 0}} \quad \bar{Y}_{1}^{\mathrm{TM}}=\frac{\varepsilon_{r 1}}{\widehat{k}_{z 1}}
$$

and (9) can be approximated as

$$
\begin{gathered}
2 \pi \widehat{b}\left(2 n_{1}^{2}-\delta^{2}\right)^{2}+j \bar{B}_{f} \pi \widehat{b}\left(2 n_{1}^{2}-\delta^{2}\right)^{2}\left(2-\delta^{2}\right) \\
-j 2 \varepsilon_{r 1} n_{1}^{2}\left(2-\delta^{2}\right)\left(1+2 \pi^{2} \widehat{b}^{2} \delta^{2}\right)=0 .
\end{gathered}
$$

By neglecting all the terms $o\left(\delta^{4}\right),(21)$ is rewritten as

$$
\begin{aligned}
& \left(4 n_{1}^{2} \pi \widehat{b}+j 4 n_{1}^{2} \bar{B}_{f} \pi \widehat{b}-j 2 \varepsilon_{r 1}\right) \\
& +\left(j \varepsilon_{r 1}-4 \pi \widehat{b}-j 4 \bar{B}_{f} \pi \widehat{b}-j 2 n_{1}^{2} \bar{B}_{f} \pi \widehat{b}-j 4 \varepsilon_{r 1} \pi^{2} \widehat{b}^{2}\right) \delta^{2}=0 .
\end{aligned}
$$

Under the optimum condition (4) (or (5)), (22) becomes

$$
\left(\frac{2 n_{1}^{2}}{\bar{B}_{f}}\right)-\left(\frac{2}{\bar{B}_{f}}+2 j+j \frac{n_{1}^{2}}{\bar{B}_{f}^{2}}\right) \delta^{2}=0,
$$

from which

$$
\begin{aligned}
\delta^{2} & =\left(\frac{2 n_{1}^{2}}{\bar{B}_{f}}\right) \frac{1}{\left(\left(2 / \bar{B}_{f}\right)+2 j+j\left(n_{1}^{2} / \bar{B}_{f}^{2}\right)\right)} \\
& =\frac{2 n_{1}^{2} \bar{B}_{f}}{2 \bar{B}_{f}+j\left(2 \bar{B}_{f}^{2}+n_{1}^{2}\right)} .
\end{aligned}
$$

For thin substrates the susceptance $\bar{B}_{f}$ is large (see (6)) so that (24) can be approximated as

$$
\begin{aligned}
\delta^{2} & \simeq \frac{2 n_{1}^{2} \bar{B}_{f}}{2 \bar{B}_{f}+j 2 \bar{B}_{f}^{2}}=\frac{n_{1}^{2}}{1+j \bar{B}_{f}}=\frac{n_{1}^{2}\left(1-j \bar{B}_{f}\right)}{1+\bar{B}_{f}^{2}} \\
& \simeq \frac{n_{1}^{2}}{\bar{B}_{f}^{2}}-j \frac{n_{1}^{2}}{\bar{B}_{f}} .
\end{aligned}
$$

By equating the real and the imaginary parts of (25) we obtain

$$
\begin{aligned}
& \delta_{R}^{2}-\delta_{J}^{2} \simeq \frac{n_{1}^{2}}{\bar{B}_{f}^{2}}, \\
& 2 \delta_{R} \delta_{J} \simeq \frac{n_{1}^{2}}{\bar{B}_{f}} .
\end{aligned}
$$

In the limit of large $\bar{B}_{f}$, the RHS of the first of (26) can be neglected with respect to the RHS of the second. Finally, from the second we obtain

$$
\delta_{R} \simeq \delta_{J} \simeq \sqrt{\frac{\varepsilon_{r 1}}{2 \bar{B}_{f}}} .
$$


Since the radiation pattern in the $E$ plane is mainly determined by radiation from TM leaky modes, a directive broadside beam is expected in the $E$ plane for sufficiently large values of the normalized shunt susceptance $\bar{B}_{f}$. Alternatively, (27) can be written in terms of the normalized thickness as

$$
\widehat{\beta}^{\mathrm{TM}} \simeq \widehat{\alpha}^{\mathrm{TM}} \simeq \sqrt{\pi \varepsilon_{r 1} \widehat{b}}
$$

which shows that a directive broadside beam in the $E$ plane requires very thin substrates.

\section{Pencil-Beam Radiation}

Based on the above analysis a fan shape of the radiation pattern can be expected, directive in the $E$ plane and nondirective in the $H$ plane. In fact, as it will be shown in Section 6 , when the antenna is designed for maximum broadside power density the pattern in the $H$ plane is almost constant. Since the pattern in (1) is of $\mathrm{BOR}_{1}$ type [21], that is,

$$
\mathbf{E}(r, \theta, \phi)=E_{0}\left[\sin \phi C_{E}(\theta) \boldsymbol{\theta}_{0}+\cos \phi C_{H}(\theta) \boldsymbol{\phi}_{0}\right],
$$

this is equivalent to saying that $C_{E}(\theta)$ is peaked and $C_{H}(\theta)$ is almost constant. Under this condition, it is easy to show that the maximum directivity of the pattern cannot be larger than 2:

$$
\begin{aligned}
D_{\max } & =\frac{2 \pi r^{2}|\mathbf{E}(r, 0, \phi)|^{2}}{\int_{0}^{2 \pi} \int_{0}^{\pi / 2}|\mathbf{E}(r, 0, \phi)|^{2} r^{2} \sin \theta d \theta d \phi} \\
& =\frac{2}{\int_{0}^{\pi / 2}\left[1+\left|C_{E}(\theta) / C_{E}(0)\right|^{2}\right] \sin \theta d \theta} \leq 2
\end{aligned}
$$

(e.g., for a HMD in free space, it is equal to $3 / 2$, as is well known).

If a pencil beam is desired, with narrow patterns in both principal planes, a linear array of HMD sources may be employed. Since the element pattern is directive in the $E$ plane (orthogonal to the HMD direction) and nondirective in the $H$ plane (parallel to the HMD direction), the elements of the array should be aligned along the HMD direction (i.e., in the cases considered so far, along the $x$-axis). Assuming for simplicity a uniform equispaced array with interelement spacing $d$ and $2 n+1$ elements, placed symmetrically with respect to the origin, the resulting array factor is $F(\theta, \phi)=$ $\sin [(2 n+1) \xi / 2] / \sin [\xi / 2]$, with $\xi=k_{0} d \sin \theta \cos \phi$ [22]. In this way the $E$ plane array pattern is determined by the element pattern, whereas the $H$ plane array pattern is determined by the array factor. In particular, for small $H$ plane beam widths $\theta_{3 \mathrm{~dB}}^{H}$ and a small array spacing (i.e., $k_{0} d \ll 1$ ),

$$
\theta_{3 \mathrm{~dB}}^{H} \simeq \frac{1.391}{n k_{0} d}
$$

approximately results [21]. On the other hand, since the $E$ plane element pattern is due to radiation from a TM leaky wave, using (7) the $E$ plane array beam width $\theta_{3 \mathrm{~dB}}^{E}$ can approximately be evaluated as [9]

$$
\theta_{3 \mathrm{~dB}}^{E} \simeq \sqrt{\frac{2 \varepsilon_{r 1}}{\bar{B}_{f}}}
$$

with the approximation being more accurate for small beam widths (i.e., large normalized PRS susceptances). The array parameters, that is, the number $n$ and the spacing $d$, can then be determined through (31) and (32), for example, by requiring that the $3 \mathrm{~dB}$ beam width of the array factor in the $H$ plane be equal to that of the element pattern in the $E$ plane. Finally, for narrow beams the resulting broadside directivity can be estimated as [22]

$$
D_{\max } \simeq \frac{9.9}{\left(2 \theta_{3 \mathrm{~dB}}^{E}\right)\left(2 \theta_{3 \mathrm{~dB}}^{H}\right)} \simeq 1.8 n k_{0} d \sqrt{\frac{\bar{B}_{f}}{2 \varepsilon_{r 1}}} .
$$

It is worth noting that, remaining within the class of directive planar antennas, a similar performance in terms of directivity could be achieved also with ordinary phased arrays of, for example, metal patch antennas. As already mentioned, these structures would require a significant larger overall thickness; moreover, by considering truncated structures, whereas the transverse dimensions would be comparable, for these conventional classes of antennas, a 2D instead of a 1D array would be required, with a considerably larger number of radiating elements and hence a higher cost.

\section{Results and Discussion}

In order to check the accuracy of the asymptotic expressions derived in the above sections, a grounded dielectric slab covered with a capacitive PRS consisting of a lowloss ferroelectric thin film is considered with $\varepsilon_{r 1}=2.2$ at the operating frequency $f=1 \mathrm{GHz}$. The values of the phase and attenuation constants have been numerically calculated as a function of the normalized shunt susceptance $\bar{B}_{f}$ representing the ferroelectric thin film in the transverse equivalent network. For each value of $\bar{B}_{f}$, the thickness $b$ of the slab has been calculated according to the optimization condition in (6). In particular, in Figure 3, the exact results are compared with the approximate asymptotic ones for the TM leaky mode. The asymptotic approximate expression (28) is seen to be very accurate in a wide range of values of $\bar{B}_{f}$, starting approximately from $\bar{B}_{f}=10$. As expected, no TE leaky waves are found. In Figure 4, the optimum subwavelength thickness is reported as a function of $\bar{B}_{f}$.

We then consider the same substrate with a fixed thickness $b$ equal to $\lambda_{0} / 70$ at the frequency $f=1 \mathrm{GHz}$ (i.e., $b=4.28 \mathrm{~mm}$ ); according to (5), in order to have maximum broadside radiation at $f=1 \mathrm{GHz}$ a normalized susceptance $\bar{B}_{f}=11.14$ is required, which can be obtained, for example, with a ferroelectric film having $t=2 \mathrm{mil}$ and $\varepsilon_{r 2}=6.68 \cdot 10^{3}$ [20]. In Figure 5, the dispersion curve of the TM leaky mode is reported as a function of frequency, together with the power density radiated at broadside by a horizontal magnetic dipole on the ground plane. It can be seen that, at $f=1 \mathrm{GHz}$, $\widehat{\beta}^{\mathrm{TM}} \simeq \widehat{\alpha}^{\mathrm{TM}} \simeq 0.31$ results which is the value predicted by the approximate formula (28). It can also be observed that the broadside power density is maximum at $f=1 \mathrm{GHz}$.

In order to investigate the radiation properties, radiation patterns in the $E$ and $H$ planes are reported in Figure 6 at the 


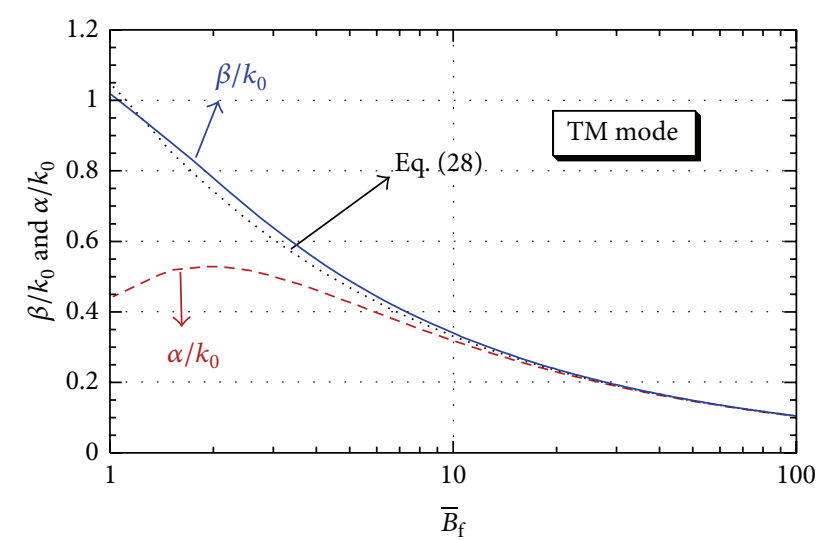

FIGURE 3: TM leaky wave normalized phase $\beta / k_{0}$ and attenuation $\alpha / k_{0}$ constants as a function of the normalized susceptance $\bar{B}_{f}$ representing the ferroelectric thin film for the proposed antenna. Parameters: $\varepsilon_{r 1}=2.2 ; f=1 \mathrm{GHz}$; for each value of $\bar{B}_{f}$, the thickness $b$ of the slab has been calculated according to the optimization condition in (6).

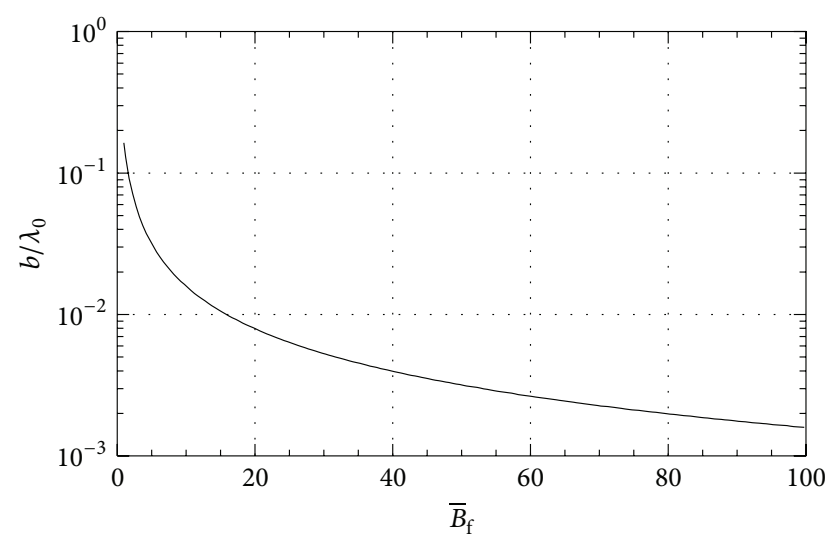

Figure 4: Optimum subwavelength thickness for directive TM radiation as a function of $\bar{B}_{f}$.

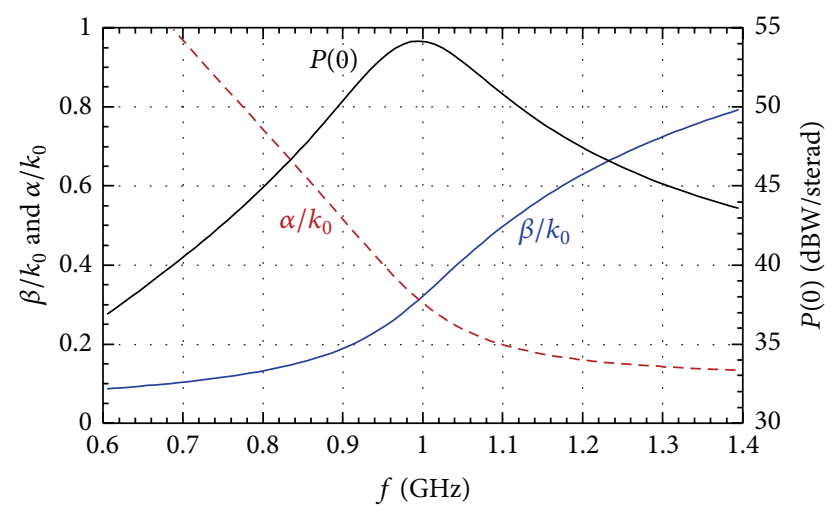

Figure 5: Dispersion curve of the supported TM leaky wave and power density radiated at broadside by a horizontal magnetic dipole on the ground plane as functions of frequency when the antenna thickness is $b=\lambda_{0} / 70$ at $f=1 \mathrm{GHz}$. Other parameters: $\bar{B}_{f}=11.14$.

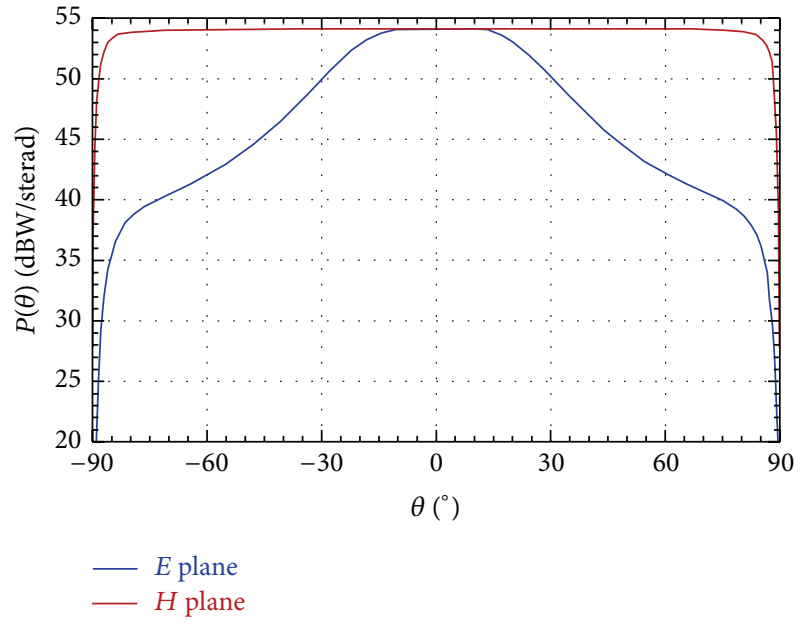

FIGURE 6: Radiation patterns in the $E$ and $H$ planes at $f=1 \mathrm{GHz}$ for a structure with $b=\lambda_{0} / 70$ and $\bar{B}_{f}=11.41$.

frequency $f=1 \mathrm{GHz}$. It can be seen that the pattern is flat in the $H$ plane (no TE leaky wave is excited), while it is quite directive (at broadside) in the $E$ plane (due to the excitation of a TM leaky wave).

More directive beams can be obtained with thinner substrates (and, accordingly, larger values of the normalized susceptance). An example is shown in Figures 7 and 8, where the same structure of Figures 5 and 6 has been considered but with a thickness $b=\lambda_{0} / 300$ at the frequency $f=1 \mathrm{GHz}$, for example, $b=1 \mathrm{~mm}$ (according to (5), a normalized susceptance $\bar{B}_{f}=47.75$ is required).

In Figure 7, the dispersion curve of the TM leaky mode is reported as a function of frequency, together with the power density radiated at broadside by a horizontal magnetic dipole over the ground plane. It can be seen that, at $f=1 \mathrm{GHz}$, $\widehat{\beta}^{\mathrm{TM}} \simeq \widehat{\alpha}^{\mathrm{TM}} \simeq 0.15$ results which is the value predicted by the approximate formula (28). It can also be observed that the broadside power density is maximum at $f=1 \mathrm{GHz}$ and, with respect to the structure of Figure 5, the broadside bandwidth has been reduced.

The radiation patterns in the $E$ and $H$ planes are reported in Figures 8(a) and 8(b) at the frequency $f=1 \mathrm{GHz}$. Again a flat pattern is radiated in the $H$ plane, whereas a highly directive beam at broadside is radiated in the $E$ plane.

In Figure 9 the patterns at different frequencies are reported in order to verify the scanning features typical of a leaky wave antenna. The radiation patterns in the principal planes are so different because in the $E$ plane the pattern is mainly determined by a weakly attenuated TM leaky wave; hence it is directive; on the other hand, as shown in Section 4, no weakly attenuated TE leaky waves exist in the considered structure at the same frequencies; hence the pattern in the $H$ plane is not directive. Please note that the radial scale in the plot of Figure 9(a) is linear (not in $\mathrm{dB}$ ); hence both the $E$ plane and the $H$ plane patterns are very broad at $0.8 \mathrm{GHz}$. (The pattern in the $H$ plane is however narrower than at $1 \mathrm{GHz}$; this may be due to the excitation at $0.8 \mathrm{GHz}$ of a TE leaky wave 


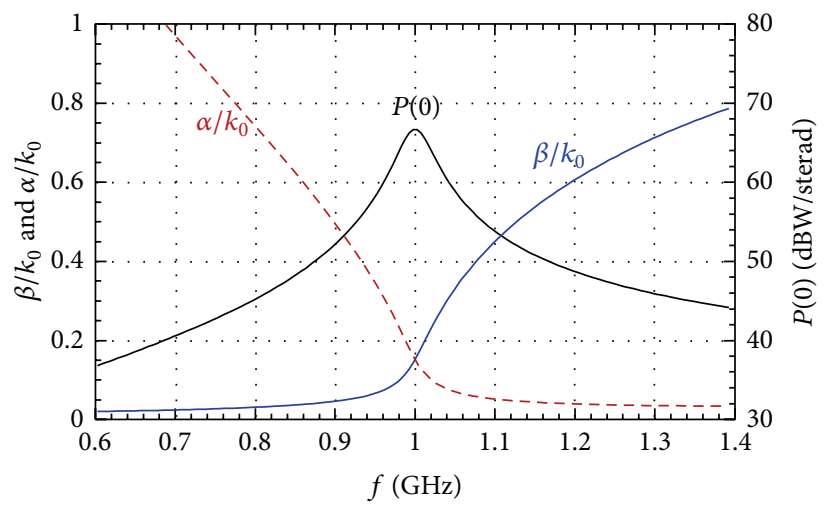

FIGURE 7: Same as in Figure 5 for a structure with $b=\lambda_{0} / 300$ at $f=1 \mathrm{GHz}$. Other parameters: $\bar{B}_{f}=47.75$.

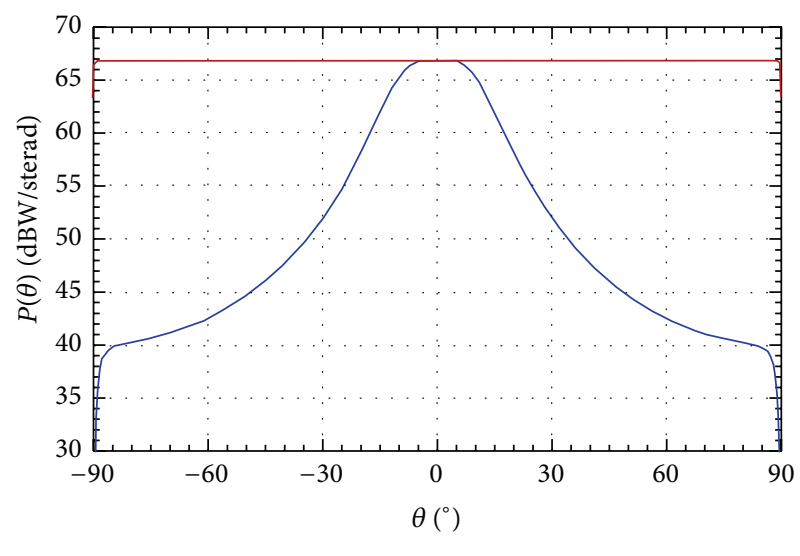

- E plane

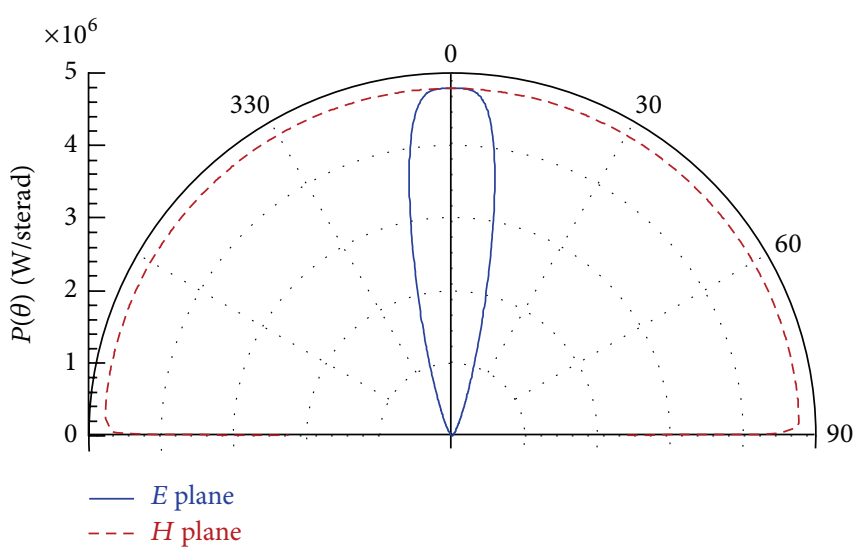

(b)

Figure 8: Radiation patterns in the $E$ and $H$ planes at $f=1 \mathrm{GHz}$ for a structure with $b=\lambda_{0} / 300$ and $\bar{B}_{f}=47.75$. Log-log plot (a) and polar linear plot (b).

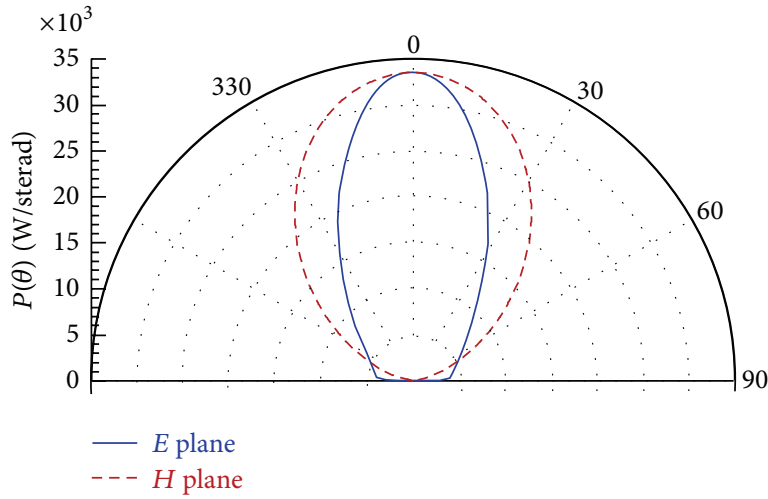

(a)

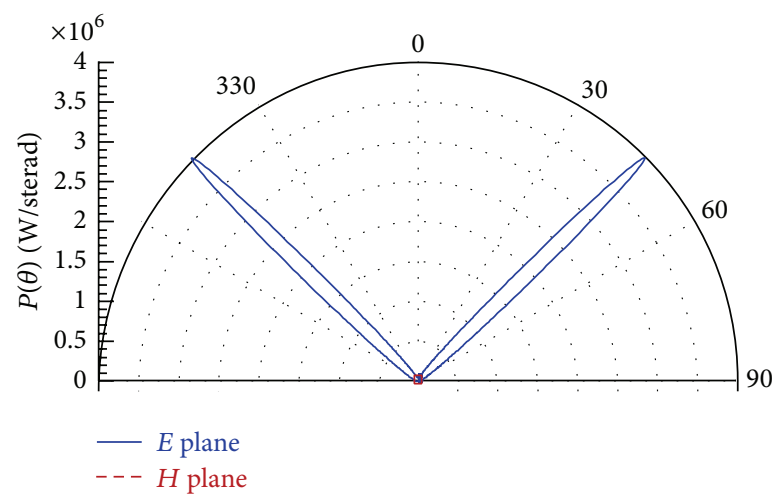

(b)

FIGURE 9: Radiation patterns in the $E$ and $H$ planes at different frequencies to show the scanning features of the proposed antenna. Parameters: $b=\lambda_{0} / 300$ at $f=1 \mathrm{GHz}$ and $\bar{B}_{f}=47.75$. (a) $f=0.8 \mathrm{GHz}$ and (b) $f=1.3 \mathrm{GHz}$. 


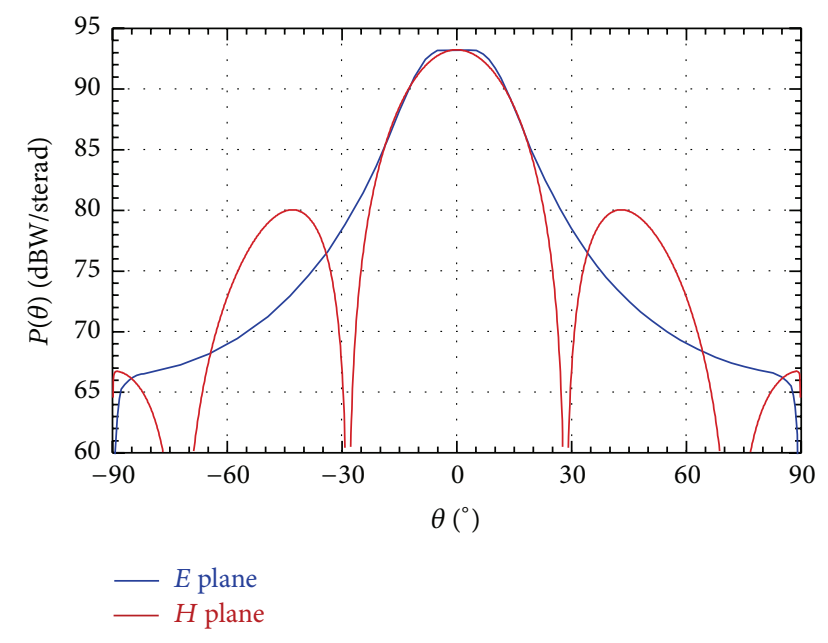

FIgURE 10: Radiation patterns in the $E$ and $H$ planes at $f=1 \mathrm{GHz}$ for a structure with $b=\lambda_{0} / 300$ and $\bar{B}_{f}=47.75$ excited by a linear array of 21 equiamplitude HMDs placed along the $x$-axis with spacing $d=\lambda_{0} / 10$.

with a large attenuation constant, which is not significant at $1 \mathrm{GHz}$ and whose existence cannot be excluded from the analysis presented in this study.) Note that in the scanned case the maximum power density in the $H$ plane is much lower than the maximum in the $E$ plane; hence the nondirective $H$ plane pattern is not visible in Figure 9(b).

The presence of losses in the ferroelectric film negligibly affects the performance of the antenna since it has been shown that doped relaxor ferroelectric materials may have very low values of the loss tangent [20].

Finally, the case of a linear array of HMD sources is considered, in order to obtain broadside pencil-beam radiation. As explained in Section 5, by aligning the sources along the HMD direction the almost isotropic pattern in the $H$ plane can be made directive; in particular, the same structure as in Figure 9 has been excited by an array of $21 \mathrm{HMD}$ elements with interelement spacing $d=\lambda_{0} / 10$, in order to obtain the same $3 \mathrm{~dB}$ beam widths in both principal planes. The relevant radiation patterns are shown in Figure 10; it can be seen that the principal-plane beam widths are indeed equal (while the secondary lobes are higher in the $H$ plane due to the uniformamplitude excitation of the array).

\section{Conclusion}

The possibility of achieving directive fan-beam radiation with planar antennas having ultrasubwavelength thickness has been demonstrated. The proposed antenna consists of highpermittivity superstrate (e.g., a low-loss ferroelectric thin film) above a ground plane; the primary source is a thin slot etched in the ground plane. In particular, it has been shown analytically that this kind of structure supports a weakly attenuated TM leaky wave which is responsible of a highly directive beam in the $E$ plane of the structure. By using a uniform array of slot sources aligned along the $H$ plane the radiation pattern can be made directive in the $H$ plane as well, thus obtaining a pencil broadside beam. Numerical results are provided which confirm the conclusion theoretically derived. Future work will concern the issues related to the practical excitation of these structures, that is, the feed design and the relevant analysis of the antenna input impedance.

\section{Conflict of Interests}

The authors declare that there is no conflict of interests regarding the publication of this paper.

\section{References}

[1] G. von Trentini, "Partially reflecting sheet arrays," IRE Transactions on Antennas and Propagation, vol. AP-4, pp. 666-671, 1956.

[2] D. R. Jackson and N. G. Alexópoulos, "Gain enhancement methods for printed circuit antennas," IEEE Transactions on Antennas and Propagation, vol. AP-33, no. 9, pp. 976-987, 1985.

[3] A. P. Feresidis and J. C. Vardaxoglou, "High gain planar antenna using optimised partially reflective surfaces," IEE Proceedings: Microwaves, Antennas and Propagation, vol. 148, no. 6, pp. 345350, 2001.

[4] T. Zhao, D. R. Jackson, J. T. Williams, H.-Y. D. Yang, and A. A. Oliner, "2-D periodic leaky-wave antennas-part I: metal patch design," IEEE Transactions on Antennas and Propagation, vol. 53, no. 11, pp. 3505-3514, 2005.

[5] T. Zhao, D. R. Jackson, and J. T. Williams, "2-D periodic leakywave antennas-part II: slot design," IEEE Transactions on Antennas and Propagation, vol. 53, no. 11, pp. 3515-3524, 2005.

[6] P. Burghignoli, G. Lovat, F. Capolino, D. R. Jackson, and D. R. Wilton, "Highly polarized, directive radiation from a fabry-prot cavity leaky-wave antenna based on a metal strip grating," IEEE Transactions on Antennas and Propagation, vol. 58, no. 12, pp. 3873-3883, 2010.

[7] D. R. Jackson and A. A. Oliner, "Leaky-wave analysis of the high-gain printed antenna configuration," IEEE Transactions on Antennas and Propagation, vol. 36, no. 7, pp. 905-910, 1988.

[8] A. Ip and D. R. Jackson, "Radiation from cylindrical leaky waves," IEEE Transactions on Antennas and Propagation, vol. 38, no. 4, pp. 482-488, 1990.

[9] G. Lovat, P. Burghignoli, and D. R. Jackson, "Fundamental properties and optimization of broadside radiation from uniform leaky-wave antennas," IEEE Transactions on Antennas and Propagation, vol. 54, no. 5, pp. 1442-1452, 2006.

[10] P. Burghignoli, G. Lovat, and D. R. Jackson, "Analysis and optimization of leaky-wave radiation at broadside from a class of 1-D periodic structures," IEEE Transactions on Antennas and Propagation, vol. 54, no. 9, pp. 2593-2604, 2006.

[11] D. R. Jackson, P. Burghignoli, G. Lovat et al., "The fundamental physics of directive beaming at microwave and optical frequencies and the role of leaky waves," Proceedings of the IEEE, vol. 99, no. 10, pp. 1780-1805, 2011.

[12] H. Ostner, J. Detlefsen, and D. R. Jackson, "Radiation from onedimensional dielectric leaky-wave antennas," IEEE Transactions on Antennas and Propagation, vol. 43, no. 4, pp. 331-339, 1995.

[13] P. Baccarelli, P. Burghignoli, F. Frezza, A. Galli, G. Lovat, and D. R. Jackson, "Approximate analytical evaluation of the continuous spectrum in a substrate-superstrate dielectric waveguide," IEEE Transactions on Microwave Theory and Techniques, vol. 50, no. 12, pp. 2690-2701, 2002. 
[14] P. Baccarelli, P. Burghignoli, F. Frezza, A. Galli, G. Lovat, and D. R. Jackson, "Uniform analytical representation of the continuous spectrum excited by dipole sources in a multilayer dielectric structure through weighted cylindrical leaky waves," IEEE Transactions on Antennas and Propagation, vol. 52, no. 3, pp. 653-665, 2004.

[15] G. Lovat, P. Burghignoli, and D. R. Jackson, "An investigation of directive radiation from ultra subwavelength-thick planar antennas with partially-reflecting surfaces," in Proceedings of the IEEE International Symposium on Antennas and Propagation and USNC/URSI National Radio Science Meeting (APSURSI '08), San Diego, Calif, USA, July 2008.

[16] G. Lovat, P. Burghignoli, and S. Celozzi, "A tunable ferroelectric antenna for fixed-frequency scanning applications," IEEE Antennas and Wireless Propagation Letters, vol. 5, no. 1, pp. 353356, 2006.

[17] T. Zhao, D. R. Jackson, J. T. Williams, and A. A. Oliner, "Simple CAD model for a dielectric leaky-wave antenna," IEEE Antennas and Wireless Propagation Letters, vol. 3, no. 1, pp. 243-245, 2004.

[18] F. de Flaviis, N. G. Alexopoulos, and O. M. Stafsudd, "Planar microwave integrated phase-shifter design with high purity ferroelectric material," IEEE Transactions on Microwave Theory and Techniques, vol. 45, no. 6, pp. 963-969, 1997.

[19] E. A. Nenasheva, N. F. Kartenko, I. M. Gaidamaka et al., "Low loss microwave ferroelectric ceramics for high power tunable devices," Journal of the European Ceramic Society, vol. 30, no. 2, pp. 395-400, 2010.

[20] Q. Zhang, J. Zhai, and L. B. Kong, "Relaxor ferroelectric materials for microwave tunable applications," Journal of Advanced Dielectrics, vol. 2, 2012.

[21] P.-S. Kildal, Foundations of Antennas, Lund, Sweden, Studentlitteratur AB, 2000.

[22] C. A. Balanis, Antenna Theory, Wiley, Hoboken, NJ, USA, 3rd edition, 2005. 

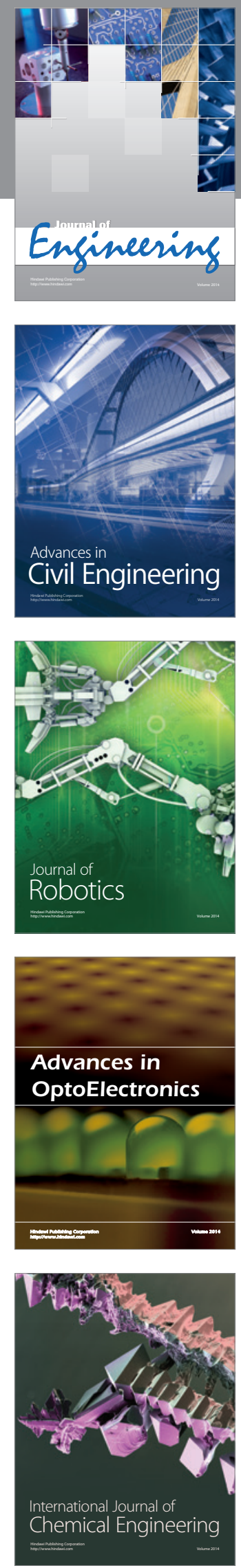

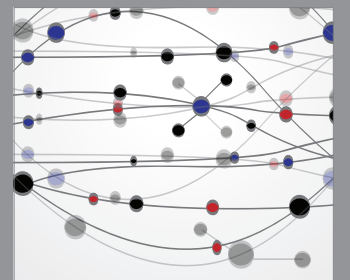

The Scientific World Journal
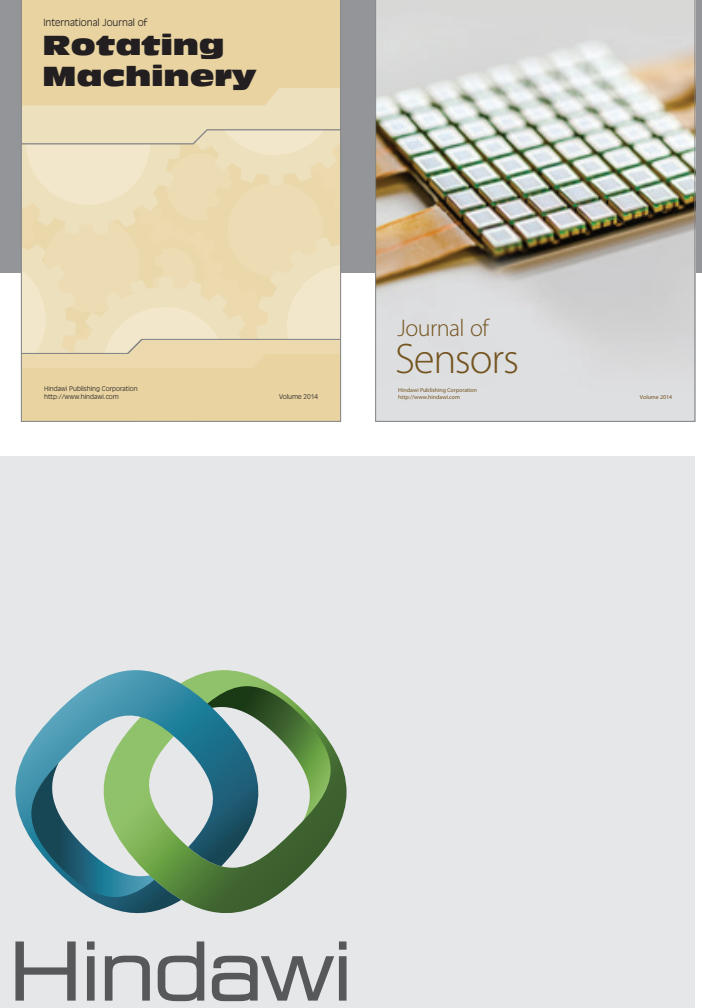

Submit your manuscripts at http://www.hindawi.com
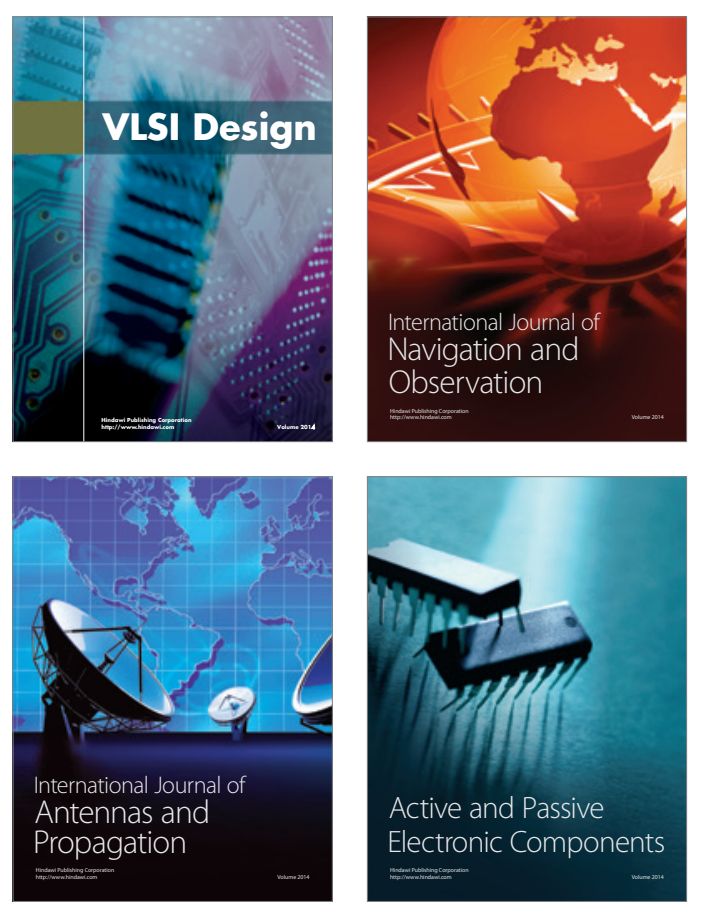
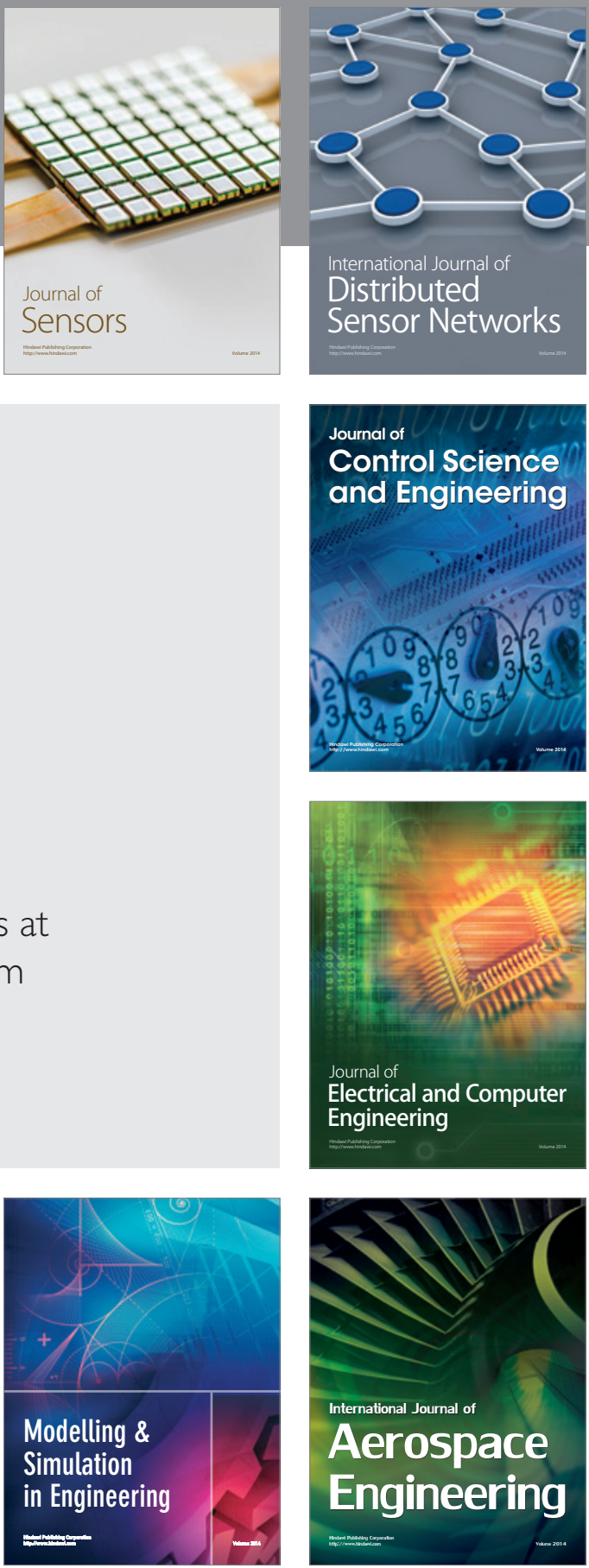

Journal of

Control Science

and Engineering
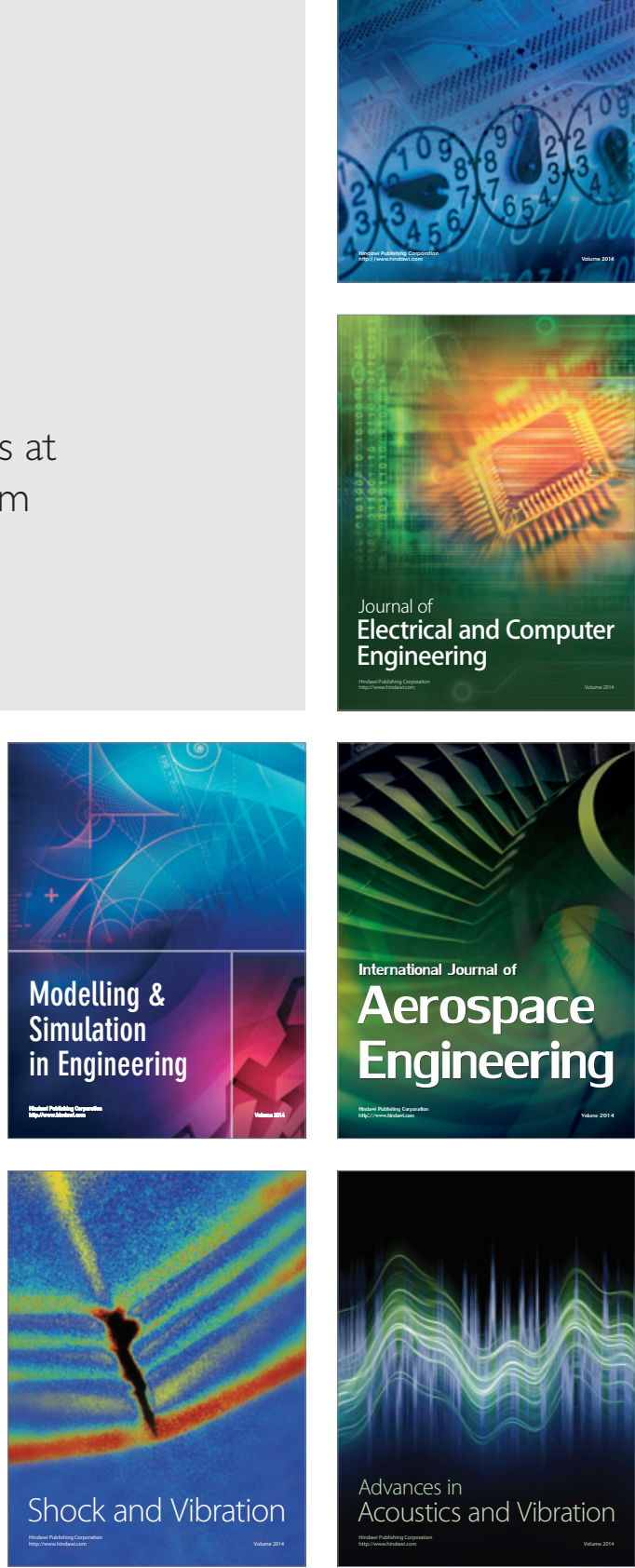